Pathologe $2017 \cdot 38: 490$

https://doi.org/10.1007/s00292-017-0386-6

Online publiziert: 9. Oktober 2017

(c) Springer Medizin Verlag GmbH 2017

CrossMark

\section{F. Fend}

Institut für Pathologie und Neuropathologie, Comprehensive Cancer Center Tübingen-Stuttgart, Universitätsklinikum Tübingen, Eberhard Karls Universität Tübingen, Tübingen, Deutschland

\title{
Tumoren der Sinnesorgane
}

Das vorliegende Schwerpunktheft beschäftigt sich mit einem Themengebiet, das an der Schnittstelle zwischen den klinischen Fächern Augen- bzw. Ohrenheilkunde und der Pathologie liegt und vielen praktizierenden Kollegen im Alltag selten begegnet. Das häufigste primäre Malignom des Auges, das maligne Aderhautmelanom, zeigt eine Inzidenz von etwa $6 / 1 \times 10^{6}$ und macht damit etwa 3-5\% aller Melanome in Westeuropa oder den USA aus. Trotz ihrer Seltenheit insgesamt weisen Tumoren der Sinnesorgane ein großes morphologisches und klinisches Spektrum auf. Manche Tumorentitäten sind aufgrund der speziellen Histogenese der Sinnesorgane nur dort nachweisbar, oder sie zeigen klinische und biologische Besonderheiten, die sie von anderen gleichartigen Tumoren anderer Lokalisation unterscheiden, und können daher diagnostisch eine Herausforderung bieten. Klinischerseits erschwert der meist vorhandene Wunsch zum Funktionserhalt und die damit verbundene Einschränkung bei der Materialgewinnung und der daraus resultierenden Quantität und Qualität vieler Gewebeproben die Diagnostik. Als Beispiel für diese Problematik kann das intraokuläre Lymphom genannt werden, das üblicherweise am Glaskörperaspirat diagnostiziert wird und bei dem degenerative Zellveränderungen und geringe Zellzahl häufig zu einer Verzögerung der Diagnosestellung führen und spezielle analytische Strategien erfordern.

Aufgrund der spezifischen klinischen Situation liegt die Diagnostik der Tumoren der Sinnesorgane nicht immer in der Hand der Pathologen, sondern wird oft von spezialisierten klinischen Kollegen durchgeführt. Dies führt einerseits auch in großen Institutionen $\mathrm{zu}$ einer mangelnden Erfahrung auf Seiten der Pa- thologie, andererseits verfügen die diagnostisch tätigen klinischen Abteilungen nicht immer über das volle methodische Spektrum der modernen Pathologie. Aus diesen Gründen ist bei der Diagnostik dieser seltenen Neoplasien unabhängig von lokalen Gegebenheiten eine enge interdisziplinäre Kooperation und Verständnis für den Blickwinkel der beteiligten Fachdisziplinen erforderlich. Obwohl die in diesem Schwerpunktheft zusammengestellten Übersichtsarbeiten keinen Anspruch auf vollständige Abdeckung des Themas „Tumoren der Sinnesorgane" stellen können, hoffen wir doch, dass die von auf dem Gebiet ausgewiesenen Experten verfassten Beiträge Verständnis für die spezielle diagnostische Problematik und Einblicke in die Biologie dieser seltenen Neoplasien liefern.

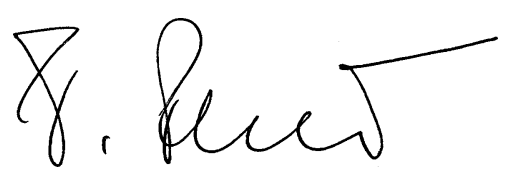

Prof. Dr. F. Fend

\section{Korrespondenzadresse}

\section{Prof. Dr. F. Fend}

Institut für Pathologie und Neuropathologie, Comprehensive Cancer Center TübingenStuttgart, Universitätsklinikum Tübingen, Eberhard Karls Universität Tübingen Liebermeisterstraße 8, 72076 Tübingen, Deutschland

falko.fend@med.uni-tuebingen.de

Interessenkonflikt. F. Fend gibt an, dass kein Interessenkonflikt besteht. 